\title{
Convergence Properties of Some Nonlinear Sequence Transformations
}

\author{
By Avram Sidi
}

\begin{abstract}
The nonlinear transformations to accelerate the convergence of sequences due to Levin are considered and bounds on the errors are derived. Convergence theorems for oscillatory and some monotone sequences are proved.
\end{abstract}

1. Introduction. Recently, Levin (1973) has developed some very powerful nonlinear transformations to accelerate the convergence of sequences (or series). These transformations have had remarkable success when applied to certain problems. For example, Levin (1973) has applied them to various infinite series, Longman (1973) has used them to generate rational approximations for Laplace transform inversion, and Blakemore, Evans, and Hyslop (1976) have used them in the computation of certain infinite integrals which come up in certain physical problems. So far, however, the convergence properties of these transformations have not been analyzed. The purpose of this paper is to partially fill this gap.

In the next section we review the derivation of the transformations of Levin. In Section 3 we give error bounds for two different limiting processes and state some sufficient conditions for convergence. The results of Section 3 are based on Sidi (1977, Chapter 5). In Section 4 the application of Levin's transformations to oscillatory and monotone sequences is considered. It turns out that for oscillatory sequences the sufficient conditions in the theorems of Section 3 are automatically satisfied, hence there is always convergence. For monotone sequences, however, in general, we do not know whether the sufficient conditions above are satisfied, and experience suggests that they are not. For some monotone sequences though we are able to give a convergence theorem. In Section 5 further convergence properties for some parameters which appear in the derivation of Levin's transformations are analyzed. In Section 6 a special case of a theorem due to Levin and Sidi (1975) is proved which shows under what conditions one could expect Levin's transformations to give convergent results.

2. Review of Levin's Transformations. Let $A_{1}, A_{2}, \ldots$ be an infinite convergent sequence whose limit we denote by $A . T_{k, n}$, the approximation to $A$, and the constants $\gamma_{i}, i=0, \ldots, k-1$, are defined as the solution to the $k+1$ linear equations

$$
A_{r}=T_{k, n}+R_{r} \sum_{i=0}^{k-1} \gamma_{i} / r^{i}, \quad r=n, n+1, \ldots, n+k,
$$

provided that no $R_{r}$ is zero. 
These equations have a simple solution for $T_{k, n}$, which is given by

$$
T_{k, n}=\frac{\sum_{j=0}^{k}(-1)^{j}\left(\begin{array}{l}
k \\
j
\end{array}\right)(n+j)^{k-1} A_{n+j} / R_{n+j}}{\sum_{j=0}^{k}(-1)^{j}\left(\begin{array}{l}
k \\
j
\end{array}\right)(n+j)^{k-1} / R_{n+j}} .
$$

The expression in (2.2) can be put in a more compact form by using forward differences. If we define $\Delta \alpha_{n}=\alpha_{n+1}-\alpha_{n}$ and $\Delta^{s} \alpha_{n}=\Delta\left(\Delta^{s-1} \alpha_{n}\right), s=2,3, \ldots$, then we have

$$
\Delta^{k} \alpha_{n}=\sum_{j=0}^{k}(-1)^{k-j}\left(\begin{array}{l}
k \\
j
\end{array}\right) \alpha_{n+j}
$$

Making use of (2.3) in (2.2), we can express $T_{k, n}$ as

$$
T_{k, n}=\frac{\Delta^{k}\left(n^{k-1} A_{n} / R_{n}\right)}{\Delta^{k}\left(n^{k-1} / R_{n}\right)} .
$$

The $t, u$, and $v$ transformations are defined by letting $R_{r}=a_{r}, R_{r}=r a_{r}$, and $R_{r}=$ $a_{r} a_{r+1} /\left(a_{r+1}-a_{r}\right)$, respectively, where $a_{1}=A_{1}, a_{r}=\Delta A_{r-1}, r \geqslant 2$. The $t$ and $u$ transformations were designed specifically for alternating and monotone series, respectively.

3. Error Bounds and Some Convergence Theorems. As is well known, in order for a certain convergence acceleration method to work well on a given sequence, the sequence in hand has to have certain properties which suit the specific convergence acceleration method. If the sequence does not have those properties, then we should not expect the method to work well. What then are the properties that the sequence $A_{r}$, $r=1,2, \ldots$, of Section 2 , should have in order for $T_{k, n}$ to be a good approximation to the limit $A$ ? Another even more important question is: Given that the sequence $A_{r}$, $r=1,2, \ldots$, has those favorable properties, how good an approximation is $T_{k, n}$ ? A partial answer to both of these questions will be given below.

Lemma. Let $T_{k, n}$ be the approximation to the limit $A$ of the sequence $A_{r}, r=$ $1,2, \ldots$, as given in (2.4). Then

$$
T_{k, n}-A=\frac{\Delta^{k}\left[n^{k-1}\left(A_{n}-A\right) / R_{n}\right]}{\Delta^{k}\left(n^{k-1} / R_{n}\right)} .
$$

Proof. Subtracting $A$ from both sides of (2.4) we obtain

$$
T_{k, n}-A=\frac{\Delta^{k}\left(n^{k-1} A_{n} / R_{n}\right)-A \Delta^{k}\left(n^{k-1} / R_{n}\right)}{\Delta^{k}\left(n^{k-1} / R_{n}\right)} .
$$

Using now the fact that $\Delta^{k}$ is a linear operator in the numerator of the expression on the right-hand side of (3.2), the result follows.

We are now going to consider two kinds of limiting processes:

1. $k$ is held fixed and $n \rightarrow \infty$, (Process I),

2. $n$ is held fixed and $k \rightarrow \infty$, (Process II). 


\section{Process I.}

THEOREM 3.1. Let the sequence $A_{r}, r=1,2, \ldots$, have the limit $A$, and let the $A_{r}$ be of the form

$$
A_{r}=A+R_{r} f(r), \quad r=1,2, \ldots,
$$

where $f(x)$, considered as a function of the continuous variable $x$, is continuous for all $x \geqslant n$, including $x=\infty$, and as $x \rightarrow \infty$, has a Poincaré-type asymptotic expansion in inverse powers of $x$, given by

$$
f(x) \sim \sum_{i=0}^{\infty} \beta_{i} / x^{i}, \text { as } x \rightarrow \infty, \beta_{0} \neq 0
$$

Define $w_{k}(x) b y$

$$
w_{k}(x)=f(x)-\sum_{i=0}^{k-1} \beta_{i} / x^{i}
$$

Then $T_{k, n}$ satisfies

$$
T_{k, n}-A=\frac{\Delta^{k}\left[n^{k-1} w_{k}(n)\right]}{\Delta^{k}\left(n^{k-1} / R_{n}\right)},
$$

where $\Delta w_{k}(x)=w_{k}(x+1)-w_{k}(x)$.

Remark. The case $\beta_{0}=0$ will be dealt with in Section 6. There we shall see that $\beta_{0} \neq 0$ is not a serious limitation.

Proof. Substituting (3.3) in (3.1), we obtain

$$
T_{k, n}-A=\frac{\Delta^{k}\left[n^{k-1} f(n)\right]}{\Delta^{k}\left(n^{k-1} / R_{n}\right)}
$$

Now, using the fact that $\Delta^{k}(p(x))=0$ whenever $p(x)$ is a polynomial in $x$ of degree at most $k-1$ and $\Delta p(x)=p(x+1)-p(x)$, we have

$$
\Delta^{k}\left(x^{k-1} \sum_{i=0}^{k-1} \beta_{i} / x^{i}\right)=0 .
$$

Subtracting the left-hand side of (3.8), with $x$ replaced by $n$, from the numerator of the right-hand side of (3.7), and using (3.5), the result follows.

COROllary 1. Define the $a_{s}^{k, n}$ by

$$
a_{s}^{k, n}=\frac{(-1)^{s}\left(\begin{array}{l}
k \\
s
\end{array}\right)(n+s)^{k-1} / R_{n+s}}{\sum_{j=0}^{k}(-1)^{j}\left(\begin{array}{l}
k \\
j
\end{array}\right)(n+j)^{k-1} / R_{n+j}}, \quad s=0,1, \ldots, k
$$

Then, $T_{k, n}$ satisfies the inequality

$$
\left|T_{k, n}-A\right| \leqslant\left(\sum_{j=0}^{k}\left|a_{j}^{k, n}\right|\right) \sup _{s \geqslant n}\left|R_{s}\right| \sup _{s \geqslant n}\left|w_{k}(s)\right| .
$$

Proof. Making use of (2.3) in (3.6) and using (3.9), (3.6) can be written as 


$$
T_{k, n}-A=\sum_{s=0}^{k} a_{s}^{k, n} R_{n+s} w_{k}(n+s) .
$$

Now since $A_{r} \rightarrow A$ and $f(x)$ is nonzero at $x=\infty$, then $R_{r} \rightarrow 0$ as $r \rightarrow \infty$. Hence, $\bar{R}_{n}=\sup _{s \geqslant n}\left|R_{s}\right|$ exists and is finite. Similarly, $\bar{w}_{k, n}=\sup _{s \geqslant n}\left|w_{k}(s)\right|$ also exists and is finite. The result in (3.10) now follows by taking the absolute value of both sides and using the inequality

$$
\left|\sum_{i=1}^{k} p_{i} q_{i}\right| \leqslant \max _{1 \leqslant i \leqslant k}\left|p_{i}\right|\left(\sum_{j=1}^{k}\left|q_{j}\right|\right) .
$$

COROllary 2. $T_{k, n}$ satisfies the inequality

$$
\left|T_{k, n}-A\right| \leqslant\left(\sum_{j=0}^{k}\left|a_{j}^{k, n}\right|\right) \eta_{k, n},
$$

where $\eta_{k, n}=o\left(n^{-k}\right)$ as $n \rightarrow \infty$; and if

$$
\sup _{n}\left(\sum_{j=0}^{k}\left|a_{j}^{k, n}\right|\right)<\infty,
$$

then, as $n \rightarrow \infty$,

$$
T_{k, n}=A+o\left(n^{-k}\right) .
$$

Proof. Since $R_{r} \rightarrow 0$ as $r \rightarrow \infty, \bar{R}_{n} \rightarrow 0$ as $n \rightarrow \infty$, hence $\bar{R}_{n}=o(1)$. Similarly, using (3.4) in (3.5), we have that

$$
w_{k}(x) \sim \sum_{i=k}^{\infty} \beta_{i} / x^{i} \text { as } x \rightarrow \infty,
$$

hence $w_{k}(x)=O\left(x^{-k}\right)$; therefore, $\bar{w}_{k, n}=O\left(n^{-k}\right)$ as $n \rightarrow \infty$. Defining now $\eta_{k, n}=$ $\bar{R}_{n} \bar{w}_{k, n}$, the results in (3.13) and (3.15) follow easily.

Process II.

THEOREM 3.2. Let the sequence $A_{r}, r=1,2, \ldots$, be as in Theorem 3.1. Using the transformation $\xi=n / x$, map the infinite interval $n \leqslant x \leqslant \infty$ to the finite interval $0 \leqslant \xi \leqslant 1$. Define $F(\xi) \equiv f(x)$ and let

$$
F_{k}(\xi)=\sum_{i=0}^{k-1} c_{k, i} \xi^{i}
$$

be the best polynomial approximation of degree $k-1$ to $F(\xi)$ on the interval $[0,1]$; define also $f_{k}(x) \equiv F_{k}(\xi)$. Further, let

$$
z_{k}(x)=f(x)-f_{k}(x),
$$

and define $Z_{k}(\xi) \equiv z_{k}(x)$. Then

$$
T_{k, n}-A=\frac{\Delta^{k}\left[n^{k-1} z_{k}(n)\right]}{\Delta^{k}\left(n^{k-1} / R_{n}\right)},
$$

where $\Delta z_{k}(x)=z_{k}(x+1)-z_{k}(x)$. 
Proof. Since $f(x)$ is continuous for all $x \geqslant n$, including $x=\infty, F(\xi)$ is continuous on $[0,1]$; therefore, best polynomial approximations of all orders to $F(\xi)$ exist on $[0,1]$; in particular, $F_{k}(\xi)$ exists there. Now

$$
f_{k}(x)=\sum_{i=0}^{k-1} c_{k, i} n^{i} / x^{i}
$$

therefore, $x^{k-1} f_{k}(x)$ being a polynomial of degree $k-1$ in $x$, we have, as in the proof of Theorem 3.1,

$$
\Delta^{k}\left[x^{k-1} f_{k}(x)\right]=0 .
$$

Subtracting now the left-hand side of (3.21), with $x$ replaced by $n$, from the numerator of the right-hand side of (3.7) again, and using (3.18), the result follows.

Corollary 1. $T_{k, n}$ satisfies the inequality

$$
\left|T_{k, n}-A\right| \leqslant\left(\sum_{j=0}^{k}\left|a_{j}^{k, n}\right|\right) \sup _{s \geqslant n}\left|R_{s}\right| \sup _{s \geqslant n}\left|z_{k}(s)\right| .
$$

The proof of this corollary is similar to that of Corollary 1 of Theorem 3.1 and will be omitted.

COROLlARY 2. If $f(x)$, in addition to being continuous, is also infinitely differentiable for $x \geqslant n$, including $x=\infty$, then $T_{k, n}$ satisfies the inequality

$$
\left|T_{k, n}-A\right| \leqslant\left(\sum_{j=0}^{k}\left|a_{j}^{k, n}\right|\right) \epsilon_{k},
$$

where $\epsilon_{k} \rightarrow 0$ as $k \rightarrow \infty$, more rapidly than any negative power of $k$; and if

$$
\sup _{k}\left(\sum_{j=0}^{k}\left|a_{j}^{k, n}\right|\right)<\infty
$$

then as $k \rightarrow \infty$,

$$
T_{k, n}=A+o\left(k^{-\lambda}\right)
$$

for any $\lambda>0$, as $k \rightarrow \infty$.

Proof. Since $f(x)$ is infinitely differentiable for $x \geqslant n$ including $x=\infty, F(\xi)$ is infinitely differentiable for $0 \leqslant \xi \leqslant 1$. As is known from the theory of best polynomial approximations, $\zeta_{k}=\max _{0 \leqslant \xi \leqslant 1}\left|Z_{k}(\xi)\right|$, as $k \rightarrow \infty$, tends to zero more rapidly than any negative power of $k$. Now

$$
\sup _{s \geqslant n}\left|z_{k}(s)\right|=\sup _{s \geqslant n}\left|Z_{k}(n / s)\right| \leqslant \max _{0 \leqslant \xi \leqslant 1}\left|Z_{k}(\xi)\right|=\zeta_{k} .
$$

Setting $\epsilon_{k}=\bar{R}_{n} \xi_{k}$, where $\bar{R}_{n}=\sup _{s \geqslant n}\left|R_{s}\right|$ as in Corollary 1 to Theorem 3.1 (3.23) follows from (3.22). Now, using (3.24) in (3.23), (3.25) follows easily.

4. Some Special Cases. In Corollary 2 to Theorem 3.1 and also in Corollary 2 to Theorem 3.2 the conditions (3.14) and (3.24) are sufficient for convergence. When Process I and Process II are viewed as summability methods, by the Silverman-Toeplitz theorem (see Powell and Shah (1972, pp. 23-27)), these conditions are necessary (but not sufficient) for both processes to be regular summability methods. It is clear that 
these conditions can be weakened by assuming that $\Sigma_{j=0}^{k}\left|a_{j}^{k, n}\right|$ grows less rapidly than $1 / \eta_{k, n}$ as $n \rightarrow \infty$ and than $1 / \epsilon_{k}$ as $k \rightarrow \infty$. However, in certain cases convergence does take place in spite of $\Sigma_{j=0}^{k}\left|a_{j}^{k, n}\right|$ growing faster than $1 / \eta_{k, n}$ and $1 / \epsilon_{k}$. An example of this will be given below. Although it is not easy to see how $\Sigma_{j=0}^{k}\left|a_{j}^{k, n}\right|$ behaves as $n \rightarrow \infty$ or $k \rightarrow \infty$ for general $R_{r}$, in one instance at least, the convergence of $T_{k, n}$ to $A$ can be proved easily, and this is done below.

THEOREM 4.1. Suppose that the sequence $A_{r}, r=1,2, \ldots$, is as described in Theorem 3.1 and in addition

$$
R_{r}=(-1)^{r}\left|R_{r}\right|, \quad r=1,2, \ldots .
$$

Then $T_{k, n}=A+o\left(n^{-k}\right)$ as $n \rightarrow \infty$. If, in addition, $f(x)$ is infinitely differentiable for $x \geqslant n$ including $x=\infty$, then $T_{k, n}=A+o\left(k^{-\lambda}\right)$, for any $\lambda>0$, as $k \rightarrow \infty$.

Proof. Using (4.1) in (3.9), we see that

$$
\left|a_{s}^{k, n}\right|=\frac{\left(\begin{array}{l}
k \\
s
\end{array}\right)(n+s)^{k-1} /\left|R_{n+s}\right|}{\sum_{j=0}^{k}\left(\begin{array}{c}
k \\
j
\end{array}\right)(n+j)^{k-1} /\left|R_{n+j}\right|}, \quad s=0,1, \ldots, k
$$

Therefore,

$$
\sum_{j=0}^{k}\left|a_{j}^{k, n}\right|=1 .
$$

Hence, the result follows from Corollary 2 of Theorem 3.1 and Corollary 2 of Theorem 3.2 .

It has been shown by Levin (1973) that for an oscillatory convergent sequence $A_{n}=$ $\sum_{i=1}^{n}(-1)^{i-1} a_{i}$ with $a_{i}>0, a_{1}>a_{2}>\cdots$, and $\lim _{n \rightarrow \infty} a_{n}=0$, the $t$ - and $u$-transformations, both in Process I and in Process II, satisfy all the conditions of the SilvermanToeplitz theorem (see Powell and Shah (1972, pp. 23-27)) and, hence, are regular.

Therefore, $T_{k, n} \rightarrow A$. Now for the $t$-transformation, which has been designed specifically for oscillatory sequences, $R_{r}=(-1)^{r-1} a_{r}$. Hence, we see from Theorem 4.1 that the condition $a_{1}>a_{2}>\cdots$ is not necessary for convergence.

Another instance in which the convergence of $T_{k, n}$ to $A$ as $n \rightarrow \infty$ (Process I) can be shown is that of some monotone sequences. This we give in the following theorem.

THEOREM 4.2. Suppose the sequence $A_{r}, r=1,2, \ldots$, is as described in Theorem 3.1. If, in addition, $R_{r}$ are all of the same sign as $r \rightarrow \infty$, and

$$
R_{r} \sim \sum_{i=0}^{\infty} \frac{\delta_{i}}{r^{\sigma+i}} \quad \text { as } r \rightarrow \infty, \sigma>0,
$$

where the right-hand side of (4.4) is a Poincaré-type asymptotic expansion, then $T_{k, n}$ $\rightarrow A$ such that

$$
T_{k, n}-A=O\left(n^{-k-\sigma}\right) \text { as } n \rightarrow \infty .
$$

Remark. $\sigma>0$ is necessary for $R_{r} \rightarrow 0$ as $r \rightarrow \infty$.

Proof. In Eq. (3.6) of Theorem 3.1, $n^{k-1} w_{k}(n)$ and $n^{k-1} / R_{n}$, by (4.4), have 
Poincaré-type asymptotic expansions in inverse powers of $n$. In fact, $n^{k-1} w_{k}(n)=$ $O\left(n^{-1}\right)$ and $n^{k-1} / R_{n}=O\left(n^{k-1+\sigma}\right)$ as $n \rightarrow \infty$. Therefore, $\Delta^{k}\left[n^{k-1} w_{k}(n)\right]=O\left(n^{-k-1}\right)$ and $\Delta^{k}\left(n^{k-1} / R_{n}\right)=O\left(n^{-1+\sigma}\right)$ as $n \rightarrow \infty$. The result now follows from (3.6).

In spite of the result in (4.5), Corollary 2 of Theorem 3.1 does not apply to this case as is shown below.

THEOREM 4.3. When the sequence $A_{r}, r=1,2, \ldots$, is as in Theorem 4.2 Process $\mathrm{I}$ is not a regular summability method.

Proof. It is enough to show that $\sum_{j=0}^{k}\left|a_{j}^{k, n}\right|$ is not bounded as $n \rightarrow \infty$. Now

$$
\sum_{j=0}^{k}\left|a_{j}^{k, n}\right|=\frac{\sum_{j=0}^{k}\left(\begin{array}{l}
k \\
j
\end{array}\right)(n+j)^{k-1} /\left|R_{n+j}\right|}{\left|\Delta^{k}\left(n^{k-1} / R_{n}\right)\right|}>\frac{(n+k)^{k-1} /\left|R_{n+k}\right|}{\left|\Delta^{k}\left(n^{k-1} / R_{n}\right)\right|} .
$$

From the proof of Theorem 4.2 we have $\Delta^{k}\left(n^{k-1} / R_{n}\right)=O\left(n^{-1+\sigma}\right)$ as $n \rightarrow \infty$. Since $(n+k)^{k-1} /\left|R_{n+k}\right|=O\left(n^{k-1+\sigma}\right)$, we can see that the right-hand side of the inequality in (4.6) is $O\left(n^{k}\right)$ as $n \rightarrow \infty$. Therefore, $\Sigma_{j=0}^{k}\left|a_{j}^{k, n}\right| \rightarrow \infty$ as $n \rightarrow \infty$ and the result follows.

For the monotone sequences as given in Theorem 4.2, we have not been able to obtain results for Process II comparable to the ones presented for Process I. However, for one case it is quite easy to prove the following:

THEOREM 4.4. If $R_{r}=r^{-1}, r=1,2, \ldots$, then Process II is not a regular summability method.

Proof. Again, all we need to show is that $\sum_{j=0}^{k}\left|a_{j}^{k, n}\right|$ is not bounded as $k \rightarrow \infty$. Now

$$
\sum_{j=0}^{k}\left|a_{j}^{k, n}\right|=\frac{\sum_{j=0}^{k}\left(\begin{array}{c}
k \\
j
\end{array}\right)(n+j)^{k}}{\left|\Delta^{k}\left(n^{k}\right)\right|}=\frac{\sum_{j=0}^{k}\left(\begin{array}{c}
k \\
j
\end{array}\right)(n+j)^{k}}{k !}>\frac{(n+k)^{k}}{k !}
$$

The right-hand side of the inequality in (4.7), by using Stirling's formula, $k ! \sim$ $k^{k} e^{-k} \sqrt{2 \pi k}$ as $k \rightarrow \infty$, is $O\left(e^{k}\right)$ as $k \rightarrow \infty$. Therefore, $\sum_{j=0}^{k}\left|a_{j}^{k, n}\right| \rightarrow \infty$ as $k \rightarrow \infty$, and the result follows.

5. Further Results. Until now we have been concerned solely with the approximation $T_{k, n}$. Now we want to investigate the $\gamma$ 's in Eqs. (2.1). First of all, they can be computed easily without having to solve Eqs. (2.1) as is shown below. As a matter of convenience, we shall write $T$ for $T_{k, n}$.

THEOREM 5.1. The $\gamma$ 's in Eqs. (2.1) can be computed recursively by using the formulas

$$
\Delta^{k}\left[n^{k+i}\left(A_{n}-T\right) / R_{n}\right]=\sum_{j=0}^{i} \gamma_{j} \Delta^{k}\left(n^{k+i-j}\right), \quad i=0,1, \ldots, k-1 .
$$

Remark. If we set $i=-1$ in (5.1), we obtain $T$, as can be seen from (2.4).

Proof. Let us multiply each of the equations in (2.1) by $r^{k+i} / R_{r}, r=n, n+1$, $\ldots, n+k$. Now let us operate on the first equation $(r=n)$

$$
n^{k+i} A_{n} / R_{n}=n^{k+i} T / R_{n}+\sum_{j=0}^{k-1} \gamma_{j} n^{k+i-j}
$$


with the operator $\Delta^{k}$. Using the fact that $\Delta^{k} p(x)=0$ when $p(x)$ is a polynomial of degree $k-1$ or less, we obtain (5.1). Now assuming that $T$ has been computed (using (2.4)), we set $i=0$ in (5.1), and using $\Delta^{k} x^{k}=k$ !, we obtain

$$
\gamma_{0}=\frac{1}{k !} \Delta^{k}\left[n^{k}\left(A_{n}-T\right) / R_{n}\right]
$$

Setting $i=1$ next in (5.1) and using the values of $T$ and $\gamma_{0}$, we compute $\gamma_{1}$ from the formula

$$
\gamma_{1}=\frac{1}{k !}\left\{\Delta^{k}\left[n^{k+1}\left(A_{n}-T\right) / R_{n}\right]-\gamma_{0} \Delta^{k}\left(n^{k+1}\right)\right\}
$$

Now set $i=2$ and so forth up to $i=k-1$.

It turns out the $\gamma$ 's too have certain interesting convergence properties as numerical experiments show. It has been observed numerically that, both for Process I and Process II, $\gamma_{j} \rightarrow \beta_{j}, j=0,1, \ldots$, whenever $f(x)$ is as in Theorem 3.1. Unfortunately, it seems to be difficult to obtain meaningful results for arbitrary sequences. However, for the monotone sequences described in Theorem 4.2, and for Process I, it is possible to state an interesting convergence theorem for the $\gamma_{j}$.

THEOREM 5.2. If the sequence $A_{r}, r=1,2, \ldots$, is as described in Theorem 4.2, with the same notation, then

$$
\gamma_{i}-\beta_{i}=O\left(n^{-k+i}\right) \text { as } n \rightarrow \infty, i=0,1, \ldots, k-1 .
$$

Proof. We shall prove (5.5) by induction on $i$. Let us first put Eq. (5.1) in a more manageable form. Using Eq. (3.3), we can write (5.1) as

$$
\begin{aligned}
(A-T) \Delta^{k}\left(n^{k+i} / R_{n}\right)+\Delta^{k}\left[n^{k+i} f(n)\right]=\sum_{j=0}^{i} \gamma_{j} \Delta^{k}\left(n^{k+i-j}\right), & \\
& i=0,1, \ldots, k-1 .
\end{aligned}
$$

From (4.4) $n^{k+i} / R_{n}=O\left(n^{k+i+\sigma}\right)$, therefore $\Delta^{k}\left(n^{k+i} / R_{n}\right)=O\left(n^{i+\sigma}\right)$. Using this with (4.5) we then have

$$
(A-T) \Delta^{k}\left(n^{k+i} / R_{n}\right)=O\left(n^{-k+i}\right) \quad \text { as } n \rightarrow \infty .
$$

By (3.4) we have

Therefore

$$
n^{k+i} f(n) \sim \sum_{j=0}^{\infty} \beta_{j} n^{k+i-j} \quad \text { as } n \rightarrow \infty
$$

$$
\Delta^{k}\left[n^{k+i} f(n)\right]=\sum_{j=0}^{i} \beta_{j} \Delta^{k}\left(n^{k+i-j}\right)+O\left(n^{-k-1}\right) \quad \text { as } n \rightarrow \infty .
$$

Combining (5.7) and (5.8), Eq. (5.6) becomes

$$
\sum_{j=0}^{i}\left(\gamma_{j}-\beta_{j}\right) \Delta^{k}\left(n^{k+i-j}\right)=O\left(n^{-k+i}\right) \quad \text { as } n \rightarrow \infty, i=0,1, \ldots, k-1 \text {. }
$$

Now let us set $i=0$ in (5.9). We obtain

$$
k !\left(\gamma_{0}-\beta_{0}\right)=O\left(n^{-k}\right) \text { as } n \rightarrow \infty,
$$


so (5.5) is true for $i=0$. Next let us assume that (5.5) is true for $i=0,1, \ldots, m-1$, $m<k$. Then for $i=m(5.9)$ gives

(5.11) $\gamma_{m}-\beta_{m}=-\frac{1}{k !} \sum_{j=0}^{m-1}\left(\gamma_{j}-\beta_{j}\right) \Delta^{k}\left(n^{k+m-j}\right)+O\left(n^{-k+m}\right) \quad$ as $n \rightarrow \infty$.

Using now the induction hypothesis that $\gamma_{j}-\beta_{j}=O\left(n^{-k+j}\right), 0 \leqslant j \leqslant m-1$, together with $\Delta^{k}\left(n^{k+m-j}\right)=O\left(n^{m-j}\right)$ as $n \rightarrow \infty,(5.11)$ becomes

$$
\gamma_{m}-\beta_{m}=O\left(n^{-k+m}\right) \text { as } n \rightarrow \infty,
$$

and this proves the theorem.

Remark. As can be seen from (5.5), the convergence of $\gamma_{i}$ to $\beta_{i}$ is strongest for $i=0\left(\gamma_{0}-\beta_{0}=O\left(n^{-k}\right)\right)$, and becomes weaker gradually as $i$ increases, and is weakest for $i=k-1\left(\gamma_{k-1}-\beta_{k-1}=O\left(n^{-1}\right)\right)$. This phenomenon has indeed been observed numerically.

6. Concluding Remarks. So far we have proved some convergence theorems for the nonlinear sequence transformations of Levin. These theorems are based mainly on the assumption that the sequence $\left\{A_{r}\right\}_{r=1}^{\infty}$ satisfies (3.3) together with (3.4). Until now, however, nothing has been said about when these conditions are satisfied. This point will be clarified by the following theorem which is a special case of a more general theorem proved by Levin and Sidi (1975).

THEOREM 6.1. Let the sequence $A_{k}=\Sigma_{r=1}^{k} a_{r}, k=1,2, \ldots$, be such that the terms $a_{r}$ satisfy a linear first order homogeneous difference equation of the form

$$
a_{r}=p(r) \Delta a_{r}, \quad r=1,2, \ldots,
$$

where $p(x)$, considered as a function of the continuous variable $x$, as $x \rightarrow \infty$, has $a$ Poincaré-type asymptotic expansion in inverse powers of $x$, of the form

$$
p(x) \sim x^{i}\left(p_{0}+\frac{p_{1}}{x}+\frac{p_{2}}{x^{2}}+\cdots\right),
$$

for $i$ an integer $\leqslant 1$. Let $\lim _{r \rightarrow \infty} A_{r}=A, A$ finite. Assume

and

$$
\lim _{r \rightarrow \infty} p(r) a_{r}=0
$$

$$
l \bar{p} \neq 1, \quad l=-1,1,2,3, \ldots,
$$

where $\bar{p}=\lim _{x \rightarrow \infty} p(x) / x$. Then $A-A_{R-1}$, as $R \rightarrow \infty$, has an asymptotic expansion of the form

$$
A-A_{R-1}=\sum_{r=R}^{\infty} a_{r} \sim a_{R} R^{i}\left(\beta_{0}+\frac{\beta_{1}}{R}+\frac{\beta_{2}}{R^{2}}+\cdots\right) .
$$

Remark. For monotonic sequences, it turns out usually that $i=1$. This is exactly what is given in the $u$-transformation of Levin, which is designed for monotonic sequences. For oscillatory sequences on the other hand it turns out usually that $i \leqslant 0$. The $t$-transformation of Levin, which is good for oscillatory sequences, has $i=0$. 
Since the proof of Theorem 6.1 is by construction, it provides us with a method for finding the asymptotic expansion in (6.5), we give it below.

Proof of Theorem 6.1. Using (6.1) in $A-A_{R-1}$, we can write

$$
A-A_{R-1}=\sum_{r=R}^{\infty} a_{r}=\sum_{r=R}^{\infty} p(r) \Delta a_{r} .
$$

Making use of the formula for "summation by parts"

$$
\sum_{r=R}^{s} g_{r} \Delta h_{r}=-g_{R-1} h_{R}+g_{s} h_{s+1}-\sum_{r=R}^{s}\left(\Delta g_{r-1}\right) h_{r},
$$

and the condition (6.3), Eq. (6.6) becomes

$$
\sum_{r=R}^{\infty} a_{r}=-p(R-1) a_{R}-\sum_{r=R}^{\infty} a_{r} \Delta p(r-1) .
$$

Now, from (6.2) and the fact that $i \leqslant 1$ and the definition of $\bar{p}$, we have

$$
p(r) \sim \bar{p} r+\bar{p}_{0}+\frac{\bar{p}_{1}}{r}+\frac{\bar{p}_{2}}{r^{2}}+\cdots, \quad \text { as } r \rightarrow \infty .
$$

Therefore,

$$
\Delta p(r-1)=\bar{p}+c_{1}(r), \quad c_{1}(r)=O\left(r^{-2}\right) \quad \text { as } r \rightarrow \infty .
$$

Substituting (6.10) into (6.8), defining $\alpha_{1}=\bar{p}+1$ and using (6.4) with $l=-1$ (hence $\left.\alpha_{1} \neq 0\right)$, we can write

$$
\sum_{r=R}^{\infty} a_{r}=d_{1}(R) a_{R}+\sum_{r=R}^{\infty} b_{1}(r) a_{r}
$$

where

$$
d_{1}(R)=-\alpha_{1}^{-1} p(R-1), \quad b_{1}(r)=-\alpha_{1}^{-1} c_{1}(r)
$$

Since $d_{1}(R) \propto p(R-1)$ and $p(R)=O\left(R^{i}\right)$ as $R \rightarrow \infty, d_{1}(R)=O\left(R^{i}\right)$ too. Similarly, since $c_{1}(r)=O\left(r^{-2}\right)$ as $r \rightarrow \infty$, we have $b_{1}(r)=O\left(r^{-2}\right)$ as $r \rightarrow \infty$; hence, the series $\sum_{r=R}^{\infty} b_{1}(r) a_{r}$ converges to zero faster than $\sum_{r=R}^{\infty} a_{r}$ as $R \rightarrow \infty$.

We now apply all the steps that led to (6.11) and (6.12) to $\sum_{r=R}^{\infty} b_{1}(r) a_{r}$. Making use of (6.1) again, we can write

$$
\sum_{r=R}^{\infty} b_{1}(r) a_{r}=\sum_{r=R}^{\infty} b_{1}(r) p(r) \Delta a_{r}=\sum_{r=R}^{\infty} q(r) \Delta a_{r}
$$

where $q(r) \equiv b_{1}(r) p(r)$. Using summation by parts, again we have

$$
\sum_{r=R}^{\infty} b_{1}(r) a_{r}=-q(R-1) a_{R}-\sum_{r=R}^{\infty} a_{r} \Delta q(r-1) .
$$

Since we have

$$
q(r)=b_{1}(r) p(r) \sim b_{1}(r)\left(\bar{p} r+\bar{p}_{0}+\frac{\bar{p}_{1}}{r}+\cdots\right) \text { as } r \rightarrow \infty,
$$

where

$$
\Delta q(r-1)=\delta_{2} b_{1}(r)+c_{2}(r), \quad c_{2}(r)=O\left(r^{-3}\right) \quad \text { as } r \rightarrow \infty,
$$


Substituting (6.16) into (6.14), defining $\alpha_{2}=1+\delta_{2}$ and using (6.4) with $l=1$ (hence $\alpha_{2} \neq 0$ ), we can write

$$
\sum_{r=R}^{\infty} b_{1}(R) a_{r}=d_{2}(R) a_{R}+\sum_{r=R}^{\infty} b_{2}(r) a_{r}
$$

where

$$
d_{2}(R)=-\alpha_{2}^{-1} q(R-1), \quad b_{2}(r)=-\alpha_{2}^{-1} c_{2}(r) .
$$

Since $q(R) \propto b_{1}(R) p(R)$ and $b_{1}(R)=O\left(R^{-2}\right)$ and $p(R)=O\left(R^{i}\right)$ as $R \rightarrow \infty$, we have $d_{2}(R)=O\left(R^{i-2}\right)$ as $R \rightarrow \infty$. Similarly, $b_{2}(r)=O\left(r^{-3}\right)$ as $r \rightarrow \infty$. Therefore, the series $\Sigma_{r=R}^{\infty} b_{2}(r) a_{r}$ converges to zero faster than $\Sigma_{r=R}^{\infty} b_{1}(r) a_{r}$ as $R \rightarrow \infty$. Continuing in this manner, we can define the functions $d_{k}(R)$ and $b_{k}(r), k=3,4,5, \ldots$, such that

$$
\sum_{r=R}^{\infty} b_{k-1}(r) a_{r}=d_{k}(R) a_{R}+\sum_{r=R}^{\infty} b_{k}(r) a_{r}
$$

where $d_{k}(R)=O\left(R^{i-k}\right)$ as $R \rightarrow \infty$ and $b_{k}(r)=O\left(r^{-k-1}\right)$ as $r \rightarrow \infty$. Adding the equations (6.11), (6.18) and (6.20) with $k=3,4, \ldots, n$, we obtain

$$
\sum_{r=R}^{\infty} a_{r}=\left[\sum_{k=1}^{n} d_{k}(R)\right] a_{R}+\sum_{r=R}^{\infty} b_{n}(r) a_{r} .
$$

Since $d_{k}(R)=O\left(R^{i-k}\right)$ as $R \rightarrow \infty$, it is clear that in the asymptotic expansion

$$
\sum_{k=1}^{n} d_{k}(R) \sim R^{i}\left[\beta_{0}+\frac{\beta_{1}}{R}+\cdots+\frac{\beta_{n}}{R^{n}}+O\left(\frac{1}{R^{n+1}}\right)\right], \quad \text { as } R \rightarrow \infty,
$$

the coefficients $\beta_{0}, \ldots, \beta_{k}$ are fixed for $k<n$. Also, since $b_{n}(r)=O\left(r^{-n-1}\right)$ and $a_{r}=$ $o(1)$ as $r \rightarrow \infty$, we have

$$
\sum_{r=R}^{\infty} b_{n}(r) a_{r}=o\left(R^{-n}\right) \quad \text { as } R \rightarrow \infty .
$$

Therefore, $\sum_{r=R}^{\infty} a_{r}$ has a true Poincaré-type asymptotic expansion as given in (6.5), thus proving the theorem.

We note here that in all the numerical examples given in Levin (1973) the sequences satisfy all the conditions given in Theorem 6.1 .

Finally, the condition $\beta_{0} \neq 0$ in (3.4), Theorem 3.1, is not too restrictive and has been imposed mainly to simplify the notation and results. The results of Section 3 remain essentially the same if $\beta_{0}=0$ and so do their proofs. In general, if $\beta_{m}(m \geqslant 0)$, is the first nonzero coefficient in (3.4), then $A_{r} \rightarrow A$ as $r \rightarrow \infty$ implies $R_{r} / r^{m} \rightarrow 0$ as $r \rightarrow \infty$. Theorem 3.1 stays the same. Inequality (3.10) in Corollary 1 has to be replaced by

(3.10)' $\left|T_{k, n}-A\right| \leqslant\left(\sum_{j=0}^{k}\left|a_{j}^{k, n}\right|\right) \sup _{s \geqslant n}\left|R_{s} / s^{m}\right| \sup _{s \geqslant n}\left|s^{m} w_{k}(s)\right| \quad$ if $k>m$.

Consequently, in Corollary 2, $\eta_{k, n}=O\left(n^{-k+m}\right)$ if $k>m$, and Eq. (3.15) has to be replaced by

$$
T_{k, n}=A+o\left(n^{-k+m}\right) .
$$

The changes in Theorem 3.2 are more complicated. Equation (3.19) now reads 


$$
T_{k, n}-A=\frac{\Delta^{k}\left[n^{k-1-m} \bar{z}_{k-m}(n)\right]}{\Delta^{k}\left(n^{k-1} / R_{n}\right)},
$$

where $\bar{z}_{l}(n / \zeta)=\bar{f}(n / \zeta)-\bar{f}_{l}(n / \zeta), f_{l}(n / \zeta)$ is the best polynomial approximation (in $\left.\zeta\right)$ of degree $l-1$ to $\bar{f}(n / \zeta)$ on $[0,1]$ and $\bar{f}(x)=x^{m} f(x)$. Inequality (3.22) in Corollary 1 now reads

$$
\left|T_{k, n}-A\right| \leqslant\left(\sum_{j=0}^{k}\left|a_{j}^{k, n}\right|\right) \sup _{s \geqslant n}\left|R_{s} / s^{m}\right| \sup _{s \geqslant n}\left|\bar{z}_{k-m}(s)\right| .
$$

The results of Corollary 2, however, stay the same. Similar changes have to be made in Sections 4 and 5 , but we shall omit them.

Department of Computer Science

Technion, Israel Institute of Technology

Haifa, Israel

1. D. LEVIN (1973), “Development of non-linear transformations for improving convergence of sequences," Internat. J. Comput. Math., v. B3, pp. 371-388.

2. D. LEVIN \& A. SIDI (1975), "Two new classes of non-linear transformations for accelerating the convergence of infinite integrals and series." (Submitted.)

3. A. SIDI (1977), Ph.D. Thesis, Tel Aviv University. (Hebrew)

4. I. M. LONGMAN (1973), "On the generation of rational approximations for Laplace trans. form inversion with an application to viscoelasticity," SIAM J. Appl. Math., v. 24, pp. 429-440.

5. M. BLAKEMORE, G. A. EVANS \& J. HYSLOP (1976), "Comparison of some methods for evaluating infinite range oscillatory integrals," J. Computational Phys., v. 22, pp. 352-376.

6. R. E. POWELL \& S. M. SHAH (1972), Summability Theory and its Applications, Van Nostrand Reinhold Co., London. 